\title{
Yoga Combined With Health Education for Risk Reduction of Metabolic Syndrome: A Randomized Controlled Pilot Feasibility Study \\ Gurjeet S Birdee ${ }^{1,2 *}$, T Alp Ikizler ${ }^{3}$, Amy Wheeler $^{7,8}$, Chase Bossart ${ }^{7}$, Dolphi Wertenbaker ${ }^{7}$, Becky Gregory ${ }^{4}$, Maciej S Buchowski ${ }^{5}$, Robert A Greevy $^{6}$, Stephanie J Sohl ${ }^{1,2}$, Mallory Jorif ${ }^{1}$ and Russell L. Rothman ${ }^{1,4}$
}

${ }^{1}$ Division of General Internal Medicine and Pediatrics, Vanderbilt University Medical Center, Nashville, Tennessee, USA

${ }^{2}$ Osher Center for Integrative Medicine, Vanderbilt University Medical Center, Nashville, Tennessee, USA

${ }^{3}$ Division of Nephrology, Vanderbilt University Medical Center, Nashville, Tennessee, USA

${ }^{4}$ Diabetes Research and Training Center, Vanderbilt University Medical Center, Nashville, Tennessee, USA

${ }^{5}$ Energy Balance Laboratory, Division of Gastroenterology, Hepatology, and Nutrition, Department of Medicine Center, Nashville, Tennessee, USA

${ }^{6}$ Department of Biostatistics, Vanderbilt University Medical Center, Nashville, Tennessee, USA

${ }^{7}$ Yoga as Therapy North America, Nashville, Tennessee, USA

${ }^{8}$ California State University, California, USA

\begin{abstract}
Objective: The primary treatment for adults with risk factors for metabolic syndrome is lifestyle change, though adoption of traditional lifestyle programs is often limited. This pilot study tested the feasibility of conducting a randomized clinical trial comparing a novel targeted yoga program coupled to lifestyle education versus lifestyle education alone among adults with metabolic syndrome risk factors.

Design and methods: Patients from primary care clinic were pre-screened electronically and further screened for metabolic syndrome. Consented participants were randomized to either a 12-week yoga program with lifestyle education or lifestyle education alone. Participants in the yoga arm received weekly 30 to 45 min of face-to-face yoga instruction, followed by 30 to $45 \mathrm{~min}$ of lifestyle education, and written instructions for home yoga practice and lifestyle changes. The lifestyle education was based on (EDU) the Group Lifestyle Balance Program that is adapted from the Diabetes Prevention Program. Participants in EDU only group received a weekly standardized curriculum matched in attention and time to the yoga with EDU arm. The primary outcome was to assess the feasibility of conducting a randomized trial by assessing recruitment rate and adherence to the protocol. Secondly, we aimed to collect preliminary data on changes in cardiometabolic factors including insulin resistance (IR) as measured by Homeostatic Model Assessment of Insulin Resistance (HOMA-IR), blood pressure, fasting lipids, body weight and composition, habitual physical activity, and dietary intake from baseline to 12-week intervention.

Results: We identified 238 adults at high risk for metabolic syndrome medical records review utilizing an electronic recruitment tool. Among eligible individuals, 67 (28\%) were enrolled and $56(84 \%)$ completed the 12-week assessment. The median attendance was 8 of 12 sessions for the yoga with EDU and 9 of 12 sessions for the EDU arm. Self-reported median frequency of home practice was 2.8 (IQR 1.4) times per week among the yoga with EDU group and 3.3 (IQR 1.1) times per week among the EDU group. Participants in the yoga with EDU arm versus EDU alone demonstrated a non-significant decrease in HOMA-IR (-0.21 (S.D. 0.99) versus 0.02 (S.D. 0.74), $p=0.29$ ). Both groups had similar changes in blood pressure, lipids, weight, body composition, energy intake, and physical activity from baseline to 12 weeks.
\end{abstract}

Conclusions: It is feasible to implement yoga combined with EDU and conduct a prospective trial to assess effectiveness on cardiometabolic risk reduction in adults with metabolic syndrome.

Keywords: Metabolic syndrome; Insulin resistance; Obesity; Yoga; Mind-body therapies

\section{Introduction}

Collectively, insulin resistance (IR), hypertension (HTN), dyslipidemia, and obesity increase the risk of diabetes [1] and cardiovascular disease [2]. This cluster of risk factors has been defined as the "metabolic syndrome" (MetS) with standard criteria for diagnosis [3]. The prevalence of MetS in the U.S is $34 \%$ among adults [4]. MetS is, in large part, caused by poor health behaviors, specifically decreased physical activity and unhealthy diets, and expert guidelines prioritize lifestyle changes for treatment [5]. Successful adaption of healthy lifestyle behaviors (increased physical activity, improved diet, and/or weight loss) among individuals with MetS correlate with improved outcomes [6,7]. Based on evidence from observational studies and clinical trials, physical exercise is recommended to individuals with MetS for at least 30 minutes a day at least 5 times a week [5]. Exercise among patients with MetS in combination with dietary changes has been shown to be superior to either treatment alone [8]. While evidence exists for benefits of exercise and dietary changes, optimal treatment has not been defined. Patient adherence to most exercise and diet programs is poor [9]. Further research is needed to identify new behavioral therapeutic options and methods to activate and sustain healthy behaviors.

*Corresponding author: Gurjeet Birdee, MD, MPH, Vanderbilt University Medica Center, Vanderbilt Center for Health Services Research, Internal Medicine and Pediatrics, Suite 6000 Medical Center East, Nashville, TN 37232-8300, USA, Tel: 615-936-0584; Fax: 615-936-1269; E-mail: gurjeet.birdee@vanderbilt.edu

Received July 27, 2015; Accepted August 04, 2015; Published August 10, 2015 Citation: Birdee GS, Ikizler TA, Wheeler A, Bossart C, Wertenbaker D, et al (2015) Yoga Combined With Health Education for Risk Reduction of Metabolic Syndrome: A Randomized Controlled Pilot Feasibility Study. J Diabetes Metab 6 : 588. doi:10.4172/2155-6156.1000588

Copyright: (c) 2015 Birdee GS, et al. This is an open-access article distributed under the terms of the Creative Commons Attribution License, which permits unrestricted use, distribution, and reproduction in any medium, provided the original author and source are credited. 
Mind-body practices (MBP), such as yoga, tai chi, and qigong, are ancient forms of mind-body exercise derived from Asia. A distinguishing feature of MBP, as compared to conventional exercise, is the emphasis on the psychological, in addition to physical, effects of practice. Key psychological features to MBP are attention regulation [10] and maintaining relaxation [11] throughout practices. MBP have been growing in popularity in the West, with yoga being the most common [12]. The 2012 National Health Interview Survey identified $8.9 \%$ of adults in the U.S. who used yoga for health in the last 12 months [13]. Yoga utilizes the following core techniques: 1. postures; 2. breathing; and 3. meditation. Experienced teachers use select techniques in composed sequences personalized to the needs of a specific population and medical condition [14]. As a form of exercise, yoga has been categorized as low, moderate, and high aerobic intensity depending on the type of physical movements employed [15-17]. There have been a small number of randomized clinical trials studying the effect of MBP, including 2 of yoga, for patients with MetS suggesting clinical benefit such as improved IR and HTN [18-22]. Conclusions are limited due to methodological issues including inadequate comparison groups, poor description of mind-body intervention and rationale, and limited data on health behaviors.

This pilot study aimed to compare the metabolic and behavioral effects of a 12-week yoga program coupled to a structured evidencebased lifestyle program compared to a lifestyle program alone among adults with metabolic syndrome risk factors. Our first objective was to determine the feasibility of conducting this randomized clinical trial. Secondly, we sought to collect preliminary data on the effects of yoga on measures of cardiometabolic risk factors. We selected insulin resistance as our main cardiometabolic outcome for two reasons: 1) expert consensus of yoga therapists who suggested that yoga would alter insulin metabolism prior to other clinical markers (e.g. weight or waist circumference); 2) insulin resistance is strongly associated with risk of type 2 diabetes and other risk factors for cardiometabolic disease (e.g. obesity, sedentary behavior, diet). We hypothesized that the 12-week yoga with lifestyle education intervention would decrease insulin resistance more than a lifestyle education intervention alone. For secondary outcomes, we anticipated further improvement in other metabolic factors (blood pressure, lipids, body composition) and lifestyle (improved diet and physical activity) among the yoga group as compared to the comparison group.

\section{Methods}

\section{Design overview}

We conducted a randomized clinical pilot study comparing yoga with a lifestyle education program to a lifestyle education program alone. The research protocol was approved by the Vanderbilt Institutional Review Board. Participants willing and eligible for the study gave written consent. After baseline assessment, participants were randomized to either 12-weeks yoga with a lifestyle education intervention or to lifestyle education alone. Participants randomized to the yoga plus lifestyle education arm received 30 to 45 minutes of weekly yoga instruction, followed by 30 to 45 minutes of lifestyle education. These participants also received written instructions for home yoga practice and lifestyle changes. Participants randomized to lifestyle alone received a weekly standardized lifestyle curriculum matched in attention and time to the yoga with lifestyle arm.

\section{Study sample}

Adult, English speaking participants were recruited from the Vanderbilt Adult Primary Care Center in Nashville, Tennessee from
$6 / 2013$ to $1 / 2014$. We utilized an electronic recruitment tool, Subject Locator, which identified potential research participants from the Vanderbilt University electronic health record. Once a "match" was made with the Subject Locator tool, research study personnel performed secondary screening through medical record review and phone interview. Participants were also recruited through posted flyers and direct referrals from primary care physicians. The study was described as a lifestyle education intervention to support weight reduction, reduce blood pressure, and improve cholesterol without mentioning of mind-body practices or yoga. We originally intended to recruit 90 participants, but only recruited 67 due to financial and time constraints.

Inclusion criteria included age 18-65 years, impaired fasting glucose (100-125 mg/dl), elevated blood pressure (systolic $\geq 130$ and/or diastolic $\geq 85$ ) or diagnosis of hypertension, dyslipidemia (triglycerides $\geq 150 \mathrm{mg} / \mathrm{dL}$ and/or HDL $\leq 40$ for males; $50 \mathrm{mg} / \mathrm{dL}$ for females) and body mass index (BMI) $25 \mathrm{~kg} / \mathrm{m}^{2}$ or greater. Potential participants were excluded if on oral diabetic medication, insulin, or lipid medication, had systolic blood pressure $\geq 160$ and/or diastolic $\geq 100$, unstable cardiac disease (e.g. angina or life threatening arrhythmia), lung disease requiring oxygen supplementation at rest or with ambulation, history of dementia or cognitive impairment, uncontrolled psychiatric disorders, such as major depression or psychosis or currently participated in a mind-body practice or program.

\section{Randomization}

The study team was blinded to randomization process. Randomization occurred after baseline testing and prior to group assignment. Randomization was stratified based on age $(\geq$ or $<50$ years) and sex. Treatment assignments were generated by a permuted blocks method randomly varying block size to 2 and 4 . Assignments were sealed in numbered, opaque envelopes.

\section{Interventions}

12-week program of health education (EDU): Subjects randomized to EDU alone received a 12-weekGroup Lifestyle Balance ${ }^{\text {tm }}$ Program, which is a comprehensive lifestyle behavior change program adapted directly from the National Institutes of Health funded Diabetes Prevention Program [23]. This program has been successfully translated to different communities as an educational intervention for MetS [2427]. We implemented content from 12-core sessions to be delivered every week for 12 weeks. EDU guides participants on behavioral change including healthy eating habits and physical activity. Participants were given instructions how to monitor energy and fat intake from the beginning of the intervention. The EDU was delivered by a registered dietitian and graduate level dietetic interns who had been trained in the EDU by the registered dietician. Classes ranged from 60 to 75 minutes to match the intervention group. Classes were offered at two different times during the week to provide participants with more flexibility to attend regularly (weekday evening and weekend day).

12-week program of yoga and health education: Participants randomized to yoga and EDU received a 12-week program designed specifically for patients with MetS. Classes were offered twice a week to provide participants more flexibility to attend regularly. Classes had two components: yoga and EDU. The total class time ranged from 60 to 75 minutes matching the EDU time above.

The 12-week yoga component was devised through a process of expert consensus. An expert panel was convened consisting of three expert yoga therapists and the principal investigator (GSB) who held 
bi-monthly meetings over nine months. All three yoga therapists are advanced teachers with more than 15 years' experience teaching therapeutic yoga.

The yoga program consists of postures, breathing, and meditation based on yoga from the Krishnamacharya tradition (Viniyoga) [14]. All movements are coordinated with breathing which requires consistent attention. The 12-week program is composed of 6 serial practices to be introduced every two weeks. The yoga component of the class initially lasted 30 minutes gradually increasing to 45 minutes over the 12 weeks. The intervention was designed to provide also gradually increase in difficulty over 12 weeks. Participants were asked to practice daily at home. Home practice was guided by written instructions and drawn pictures of each class.

Four yoga teachers received advance training on how to administer the intervention with high fidelity. All yoga teachers had completed basic yoga teacher training programs accredited by Yoga Alliance, a nationally recognized organization that standardizes yoga certification. Advance training consisted of intensive workshops covering the rationale, techniques, and administration of intervention. Workshops were taught by an expert yoga therapist from the panel. Prior to the initiation of this study, we conducted a single arm pilot study of 10 participants with METS to demonstrate fidelity in administering the yoga intervention (data not presented). We demonstrated high fidelity with more than $80 \%$ of participants completing the 12 -week intervention. In addition, yoga teachers demonstrated high fidelity in delivering the intervention utilizing a checklist (more than $80 \%$ fidelity through observation of teaching).

The lifestyle education segment of the class occurred immediately after the yoga segment for 30 to 45 minutes. The lifestyle education was derived from the EDU described above. Participants received the same content and material in a condensed fashion. The EDU content was modified to encourage yoga as the primary physical activity during the study period. The same dieticians who delivered the EDU to the comparison group delivered the EDU to yoga group. During our prior pilot study, we had successfully delivered the EDU coupled with yoga to 10 participants over 12 -weeks.

\section{Outcomes}

The primary goal of this pilot study was to assess feasibility of conducting a randomized clinical study with a yoga intervention coupled to EDU. Feasibility (recruitment to study, attendance to weekly sessions, frequency of home practice, and retention rate) and outcome variables were collected during the study period. Research assistants were not blinded to assignment at 12-weeks. Baseline information consisted of sociodemographic data. At baseline and 12-weeks, the following components of metabolic syndrome were assessed: insulin resistance as measured by the homeostasis model assessment (HOMA-IR) derived from fasting glucose and insulin levels [28] (primary outcome of interest), systolic and diastolic blood pressure, fasting lipids including total cholesterol, triglycerides, HDL and calculated LDL, weight, body composition and nutritional intake (secondary outcomes). Dietary changes were assessed with the Block Brief 2000 Food Frequency Questionnaire (FFQ) [29]. Physical activity was estimated using the accelerometers (Actigraph G3M, Actigraph LLC, Pensacola, FL) worn for seven days at the two time points [30,31]. The frequency of recommended physical activity per week was assessed among both groups at weekly sessions by self-report (How many times in the past week did you [practice yoga/exercise] as recommended in the program?).

\section{Statistical analyses}

All analyses were performed as intention-to-treat. Continuous data was reported as median with interquartile range and categorical data as percentages. Baseline data were compared using a $t$ test for continuous variables and chi squared tests for categorical variables to assess randomization. To determine feasibility, we recorded number of eligible individuals approached, number who declined participation, number successfully recruited, attendance at weekly sessions, and protocol adherence Protocol adherence was documented in terms of number of participants that completed the study with 12 -week outcome assessments. We considered high protocol adherence if $80 \%$ or more of participants completed the 12-week protocol. Adverse events were systematically tracked during the study period.

To assess differences in IR, blood pressure, lipids, body composition, nutritional intake, and physical activity, we utilized a two-sample nonparametric Wilcoxon rank-sum tests to compare the distribution of change from baseline to 12 weeks between treatment groups. The range of HOMA-IR was broader and lower than anticipated. Taking this into account, we used a linear regression model to assess changes in HOMAIR by intervention group taking into account baseline HOMA values. Analyses were performed using SPSS statistical software (Version 22) and $R$ (Version 3.0.1 www.r-project.org).

Sample size calculation: The sample size was derived based on the results of a previously published single arm 12-week clinical study of t'ai chi among patients with elevated glucose that reported changes in HOMA. ${ }^{32}$ We chose this study because t'ai chi is a mind-body practice which we estimate approximates the physical intensity of our yoga program. Based on this study, we calculated the power to detect changes in IR as measured by HOMA. In the t'ai chi study, the standard deviation for change in HOMA was 0.65 and a difference of -.53 was reported. For our study, we estimated that EDU alone will demonstrate a small change of -0.10 in HOMA. Accounting for $10 \%$ loss to followup and a $10 \%$ loss in efficiency for non-parametric testing in contrast to a t-test, we estimated that a sample size of 45 patients per group would provide $80 \%$ power to detect a HOMA-IR difference of 0.43 with a 2 sided 5\% significance level between yoga with EDU versus EDU alone. However, we recruited 67 patients due to financial and time constraints.

\section{Results}

\section{Participants}

Of the 698 individuals the electronic recruitment tool identified during primary screening, 238 (34\%) were eligible based on secondary screening through medical record review and phone interviews (Figure 1). We contacted all these individuals, $67(28 \%)$ consented to participation and were followed from $9 / 2013$ through $4 / 2014$. A total of 56 participants completed the 12 week assessment with an overall follow-up rate of $84 \%$. Similar number of participants in both groups did not complete follow-up (6 participants in yoga with education and 5 participants in education only). There were no adverse events among study participants related to yoga practice for the duration of the study.

Baseline characteristics of participants were balanced across groups (Table 1). The mean $( \pm S D)$ age of participants was 58 (10) years with half men $(51 \%)$ and predominately non-Hispanic Whites (78\%). The mean $( \pm S D)$ metabolic parameters of the participants were: fasting blood glucose of 108(16) mg/dL, insulin 15.1(7.7) $\mathrm{mg} / \mathrm{dL}$, and HOMAIR $2(1.0)$. The mean BMI was $34(6) \mathrm{kg} / \mathrm{m}^{2}$ with a waist circumference of 111(12) centimeters. Two thirds of all participants were taking 


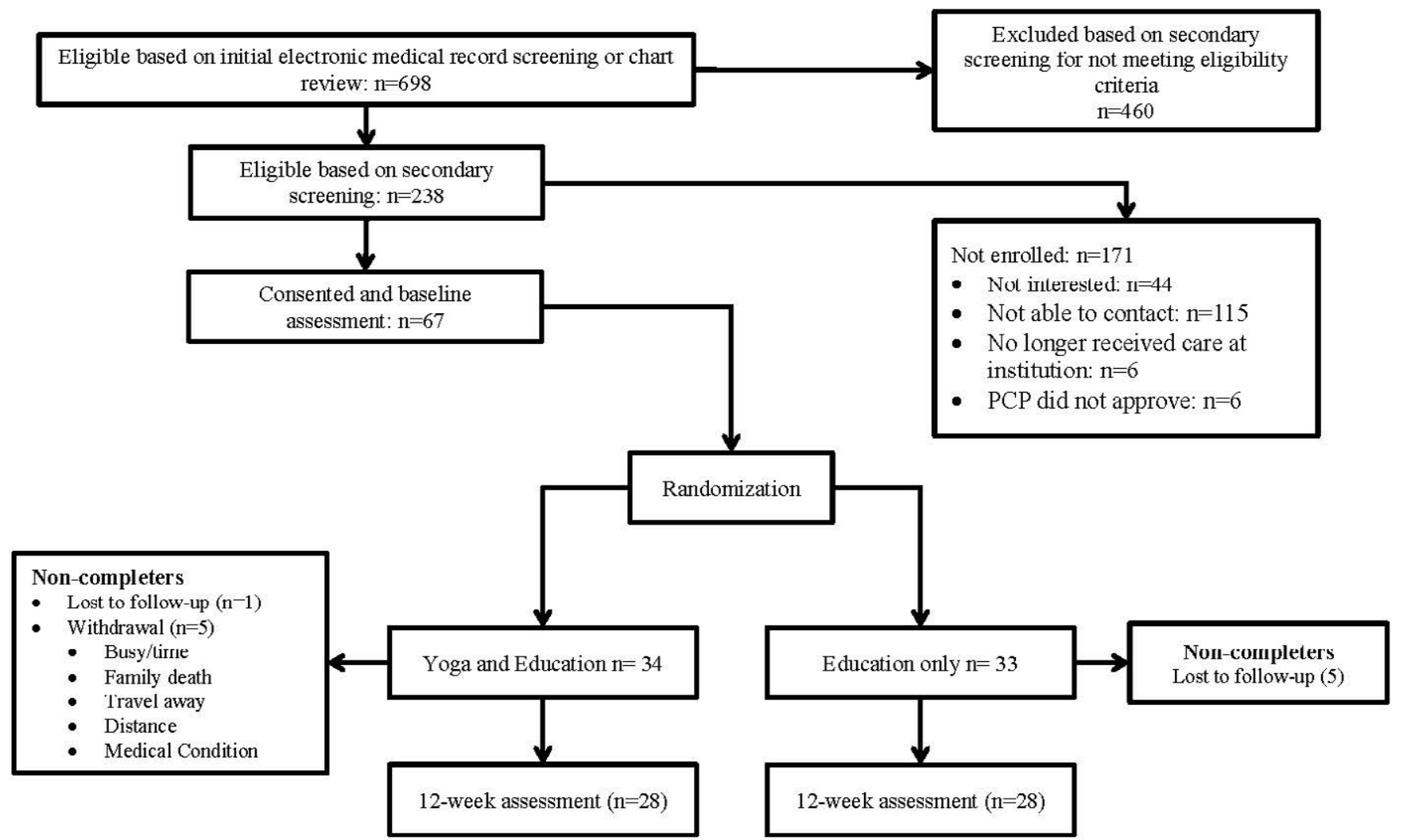

Figure 1: Flow diagram for study.

blood pressure lowering medications. The mean HDL and triglycerides were $44(10) \mathrm{mg} / \mathrm{dl}$ and $150(59) \mathrm{mg} / \mathrm{dl}$ respectively. Participants were identified as being at risk for metabolic syndrome and eligible for the study based on electronic medical record data. Among enrolled participants, $35 \%$ had all 4 criteria for metabolic syndrome at baseline data collection.

\section{Intervention adherence}

The median (interquartile range) attendance was 9 (4.0) for EDU alone versus 8 (3.0) for yoga with EDU group ( $\mathrm{p}=0.142$, Table 2). Selfreported median frequency of physical activity at home was significantly higher among EDU only versus yoga with EDU group ((3.4 times/week [1.2]) versus $2.8[1.4], \mathrm{p}=0.010)$. Five participants stopped attending ( 3 in yoga with EDU and 2 in EDU alone), but completed the 12-week assessment.

\section{Outcome measures}

As compared to the EDU alone, yoga with EDU demonstrated a non-significant reduction in insulin resistance based on HOMA-IR from baseline to 12 weeks (Table 3). The median change is shown from baseline to 12 weeks for both groups in Figure 2. In a sensitivity analysis, we examined HOMA via a multivariate linear regression model to better account for the association between baseline and 12 week HOMA. On average, larger baseline HOMA associated with greater absolute reductions 12 week HOMA ( $p=0.001,3 \mathrm{df}$ likelihood ratio test). Figure 3 shows the 12 week predicted mean difference in HOMA with 95\% confidence bands between the yoga with EDU group versus EDU group alone while accounting for baseline variations in HOMA (calculated via a parametric bootstrap with 10,000 samples).
Weight loss and percent fat mass loss (as measured by bioimpedance) was similar in both groups. Systolic blood pressure decreased slightly in the yoga group, while diastolic decreased in both groups. Some reduction in triglycerides was observed in both groups, but other lipid parameters remained similar.

Both groups substantially reduced their overall caloric intake based on the Food Frequency Questionnaire. Actigraph data were available for 45 participants (yoga with EDU=22, EDU only=23). Small increases in physical activity were observed in both groups, mostly in light physical activity. Participants did not engage in vigorous physical activity.

\section{Discussion}

In this pilot study we demonstrated feasibility of conducting a randomized clinical trial to compare yoga with EDU versus EDU alone among adults at high risk for cardiometabolic disease. Our data indicate that this is a feasible approach to implement and conduct a larger randomized clinical trial examining efficacy. We found a nonstatistically significant decrease in insulin resistance as measured by HOMA among patients receiving yoga with EDU. Changes in insulin resistance were more pronounced among patients with higher baseline insulin resistance. Both groups had similar changes in weight loss, body composition, blood pressure, and lipids. Overall, nutrition and physical activity changes were also similar among groups from baseline to 12 weeks. If a larger randomized clinical trial demonstrates that yoga significantly reduces insulin resistance more than conventional lifestyle programs, this would support the use of yoga as an adjunct therapy for individuals with elevated cardiometabolic risk. In 
Citation: Birdee GS, Ikizler TA, Wheeler A, Bossart C, Wertenbaker D, et al. (2015) Yoga Combined With Health Education for Risk Reduction of Metabolic Syndrome: A Randomized Controlled Pilot Feasibility Study. J Diabetes Metab 6: 588. doi:10.4172/2155-6156.1000588

Page 5 of 8

\begin{tabular}{|c|c|c|c|c|}
\hline Participant characteristics & All $(n=67)$ & $\begin{array}{l}\text { Educational } \\
\quad(n=33)\end{array}$ & $\begin{array}{l}\text { Yoga with Education } \\
(n=34)\end{array}$ & P-value ${ }^{1}$ \\
\hline Age in years (mean) & $58(10)$ & $57(11)$ & $59(10)$ & 0.754 \\
\hline \multicolumn{5}{|l|}{ Race (\%) } \\
\hline Non-Hispanic White & $52(78)$ & $26(79)$ & $26(77)$ & 0.357 \\
\hline Non-Hispanic Black & $13(19)$ & $7(21)$ & $6(18)$ & \\
\hline Hispanic & $2(3)$ & 0 & $2(6)$ & \\
\hline \multicolumn{5}{|l|}{ Sex (\%) } \\
\hline Male & $34(51)$ & $17(52)$ & $16(47)$ & 0.715 \\
\hline Female & $33(49)$ & $16(49)$ & $18(53)$ & \\
\hline \multicolumn{5}{|l|}{ Education } \\
\hline High school or less & $8(12)$ & $4(13)$ & $4(12)$ & 0.326 \\
\hline Some or 4 year college degree & $33(51)$ & $19(59)$ & $14(42)$ & \\
\hline More than college degree & $24(37)$ & $9(28)$ & $15(46)$ & \\
\hline \multicolumn{5}{|l|}{ Biometrics (mean, S.D.) } \\
\hline Weight (kg) & $100(17)$ & $98(17)$ & $103(17)$ & 0.273 \\
\hline Body Mass Index $\left(\mathrm{kg} / \mathrm{m}^{2}\right)$ & $34(6)$ & $33.5(6.0)$ & $33.7(6.0)$ & 0.217 \\
\hline Waist Circumference (cm) & $111(12)$ & $113(13)$ & $109(12)$ & 0.235 \\
\hline \multicolumn{5}{|l|}{ Blood pressure $(\mathrm{mm} / \mathrm{Hg})$} \\
\hline Systolic & $129(13)$ & $130(12)$ & $129(15)$ & 0.723 \\
\hline Diastolic & $76(10)$ & $77(9)$ & $76(10)$ & 0.682 \\
\hline Hypertension Medication (Yes, \%) & $44(66)$ & $23(67)$ & $21(63)$ & 0.730 \\
\hline \multicolumn{5}{|l|}{ Laboratory Values (mean, S.D.) } \\
\hline Glucose (mg/dl) & $108(16)$ & $108(19)$ & $109(12)$ & 0.333 \\
\hline Insulin (mg/dl) & $15.1(7.7)$ & $15.5(8.7)$ & $14.1(5.6)$ & 0.585 \\
\hline HOMA-Insulin Resistance & $2.0(1.0)$ & $2.1(1.1)$ & $1.9(0.7)$ & 0.581 \\
\hline \multicolumn{5}{|l|}{ Lipids } \\
\hline Total Cholesterol (mg/dl) & $198(36)$ & $192(35)$ & $202(37)$ & 0.192 \\
\hline Low density lipoprotein (LDL, mg/dL) & $123(29)$ & $118(32)$ & $128(27)$ & .065 \\
\hline High density lipoprotein (HDL (mg/dL) & $44(10)$ & $42(8)$ & $45(11)$ & .082 \\
\hline Triglycerides (TG, mg/dL) & $150(59)$ & $154(58)$ & $142(57)$ & 0.751 \\
\hline Number of criteria for metabolic syndrome ${ }^{2}(\%)$ & & & & 0.513 \\
\hline 1 & $5(8)$ & $4(12)$ & $1(3)$ & \\
\hline 2 & $21(32)$ & $10(48)$ & $11(33)$ & \\
\hline 3 & $17(26)$ & $9(53)$ & $8(24)$ & \\
\hline 4 & $23(35)$ & $10(44)$ & $12(39)$ & \\
\hline Energy intake ( kcal/day, mean, S.D.) & $1796(678)$ & $1780(708)$ & $1810(658)$ & 0.857 \\
\hline
\end{tabular}

Table 1: Baseline characteristics of participants.

\begin{tabular}{|c|c|c|c|c|}
\hline & All $(n=54)$ & EDU $(n=29)$ & Yoga + EDU (n=24) & P value ${ }^{1}$ \\
\hline Number of sessions attended (median, IQR) & $8.0(3.0)$ & $9(3.5)$ & $8(5.0)$ & 0.189 \\
\hline Total 12 weeks frequency of practice (median, IQR) & $25(16)$ & $27.0(14.5)$ & $22.0(20.5)$ & 0.018 \\
\hline Practice per week (median, IQR) & $3(1.20)$ & $3.3(1.1)$ & $2.8(1.4)$ & 0.010 \\
\hline $\begin{array}{l}\text { Stopped attending classes but completed 12-week assessment } \\
\text { (number of participants, week of drop-out) }\end{array}$ & 5 & $\begin{array}{l}\text { - Family death: } n=1 \\
\text { (Week } 0) \\
\text { - } \quad \text { Time/too busy: } n=1 \text { (Week } 3 \text { ) }\end{array}$ & $\begin{array}{l}\text { - } \quad \text { Time/too busy: } n=2 \\
\text { (Week 2, Week 3) } \\
\text { - Work: } n=1 \text { (Week 3) }\end{array}$ & N/A \\
\hline
\end{tabular}

Table 2: Intervention adherence.

particular, individuals with higher insulin resistance with higher risk for cardiometabolic disease may benefit the most from yoga practice.

The rationale for this pilot and feasibility study was to assess if participants at risk for metabolic syndrome could be enrolled, randomized, and delivered the experimental interventions. Enrolled participants were willing to be randomized and we achieved our goal of $80 \%$ or more adherence to the research protocol. While attendance to sessions was similar among groups, individuals in the yoga group with EDU reported less home practice than the EDU alone. It is unclear if the difference in home practice would produce measurable differences in metabolic outcomes. Overall physical activity was similar in both groups based on limited data available from accelerometers. Since participants in the yoga group were prescribed a specific activity 
Citation: Birdee GS, Ikizler TA, Wheeler A, Bossart C, Wertenbaker D, et al. (2015) Yoga Combined With Health Education for Risk Reduction of Metabolic Syndrome: A Randomized Controlled Pilot Feasibility Study. J Diabetes Metab 6: 588. doi:10.4172/2155-6156.1000588

Page 6 of 8

\begin{tabular}{|c|c|c|c|c|c|c|c|}
\hline & \multicolumn{2}{|c|}{ Education $(n=28)$} & \multirow[b]{2}{*}{ Difference } & \multicolumn{2}{|c|}{ Yoga with Education $(n=28)$} & \multirow[b]{2}{*}{ Difference } & \multirow[t]{2}{*}{ P value $^{1}$} \\
\hline & Baseline & 12 weeks & & Baseline & 12 weeks & & \\
\hline \multicolumn{8}{|l|}{ Obesity related } \\
\hline Weight (kg) & $101.8(26.3)$ & $100.3(24.0)$ & $-2.4(7.4)$ & $93.0(24.5)$ & $90.3(25.6)$ & $-2.5(6.5)$ & 0.831 \\
\hline Body Mass Index (kg/m2) & $34.8(7.0)$ & $33.2(9.1)$ & $-1.3(3.0)$ & $32.1(6.7)$ & $32.1(6.9)$ & $-1.2(2.3)$ & 0.818 \\
\hline Waist Circumference (cm) & $111.8(13.8)$ & $109.0(16.3)$ & $-2.0(15.6)$ & $106.0(9.0)$ & $105.0(20.6)$ & $-4.0(9.3)$ & 0.796 \\
\hline \multicolumn{8}{|l|}{ Blood pressure } \\
\hline Systolic & $128.0(14.7)$ & $128.5(16.3)$ & $2.5(24)$ & $128.5(20.8)$ & $126.0(22)$ & $-1.0(20)$ & 0.605 \\
\hline Diastolic & $78(15)$ & $74.5(16.8)$ & $-3.5(17.8)$ & $78.0(14)$ & $72.0(15.3)$ & $-3.0(7)$ & 0.780 \\
\hline \multicolumn{8}{|l|}{ Laboratory Values } \\
\hline Glucose & $103(14.5)$ & $104(13.8)$ & $0.5(13)$ & $107(15)$ & $106.0(15)$ & $2.0(13.0)$ & 0.626 \\
\hline Insulin & $12.1(9.3)$ & $11.5(9.1)$ & $-0.2(5.8)$ & $13.5(9.3)$ & $11.2(9.2)$ & $-1.6(7.1)$ & 0.539 \\
\hline HOMA & $1.64(1.17)$ & $1.53(1.54)$ & $.02(0.74)$ & $1.82(1.2)$ & $1.48(0.9)$ & $-.21(0.99)$ & 0.287 \\
\hline Cholesterol & $192.5(45.8)$ & $184.5(53.5)$ & $-6.5(25.25)$ & $191(43.3)$ & $189.0(51)$ & $-5.0(37.0)$ & 0.617 \\
\hline Low density lipoprotein & $122.5(38.3)$ & $112.0(35.0)$ & $-2.5(19.3)$ & $120.5(30.3)$ & $117.0(48)$ & $-3.0(25.0)$ & 0.496 \\
\hline High density lipoprotein & $42.0(10.8)$ & $45.5(14.3)$ & $1.0(6.5)$ & $43.0(13.8)$ & $46.0(14)$ & $1.0(9.0)$ & 0.993 \\
\hline Triglycerides & $163.5(73.8)$ & $157(104.8)$ & $-14.5(61.5)$ & $124.0(36.25)$ & $112.0(82)$ & $-13(75)$ & 0.676 \\
\hline \multicolumn{8}{|l|}{ Energy Balance } \\
\hline Dietary intake (kcal/day) & $1723(810)$ & $1345(628)$ & $-502(737)$ & $1790(378)$ & $1243(1036)$ & $-434(602)$ & 0.789 \\
\hline \multicolumn{8}{|l|}{ Physical Activity } \\
\hline $\begin{array}{l}\text { (average total counts vector } \\
\text { magnitude per day) }\end{array}$ & 345498 (159381) & $428054(198105)$ & $46119(243863)$ & $357451(220150)$ & $378252(248300)$ & $8305(176775)$ & 0.946 \\
\hline Average Kcal per day & $334(183)$ & $471(322)$ & $81(215)$ & $297(300)$ & $401(294)$ & 42 (173) & 0.296 \\
\hline
\end{tabular}

${ }^{1}$ Wilcoxon rank-sign test comparing change from baseline to 12 weeks between Education only versus Yoga with Education

Table 3: Metabolic parameters at baseline and 12 weeks by intervention group (median, IQR).

Changes in HOMA among groups from baseline to 12 weeks

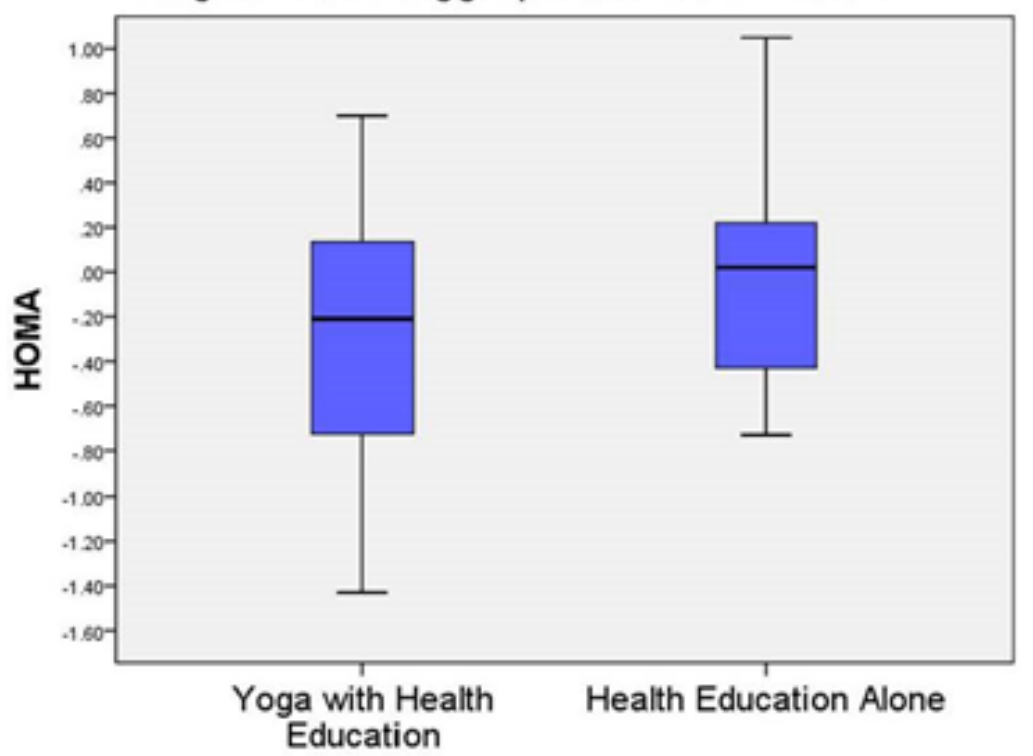

Figure 2: Changes in HOMA among groups from baseline to 12 weeks.

as compared to general exercise recommendations to the EDU alone group, it may be that participants had more difficulty with adherence.

However, several issues need to be taken into account when interpreting our results. First, we did not achieve our recruitment goal based on our a priori power analysis primarily due to financial constraints. However, had we achieved the proposed sample size of 90 participants, we still would not had sufficient power due to the standard deviation of the main metabolic outcome of interest- HOMA-IR. The standard deviation of HOMA in our study was about double that of the study by Liu et al., which we used to perform our power analysis. ${ }^{32}$
Secondly, only one third of participants in our study met all of the criteria for metabolic syndrome. All participants were at risk for cardiometabolic disease with at least 1 or more criteria for metabolic syndrome. The lower prevalence of metabolic syndrome probably resulted from our screening mechanism. We utilized electronic medical charts to identify eligible patients who met our entry criteria (impaired fasting glucose, elevated blood pressure or diagnosis of hypertension, dyslipidemia, body mass index of 25 or greater) and did not perform repeat testing for enrollment. This may have led to participants being enrolled whose values changed by the time of baseline data collection (e.g. blood pressure or blood glucose normalizing after enrollment). 


\section{IR (blue-Yoga with EDU, red-EDU alone)}

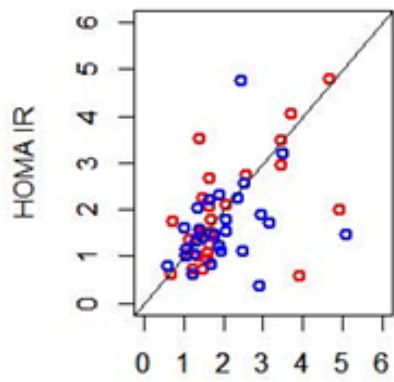

HOMA IR at baseline

\section{HOMA mean difference between treatments ( $95 \%$ C.I.)}

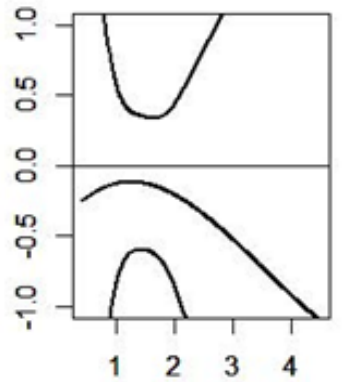

HOMA IR at baseline

Figure 3: Mean differences in HOMA-IR at 12-weeks by baseline HOMA-IR.

As our sample population had fewer participants that met all the criteria for metabolic syndrome, HOMA-IR values were lower than anticipated at baseline. As expected, HOMA-IR values were higher among participants with more risk factors for metabolic syndrome. The decreases in HOMA-IR observed among the yoga with EDU group as compared to EDU alone were largely driven by participants with high baseline HOMA-IR values. While HOMA-IR differences were not statistically significant between groups, future studies with sufficient sample of participants with higher cardiometabolic risk at baseline are necessary to determine the clinical effectiveness of yoga with EDU.

Prior clinical research has demonstrated lifestyle changes improve parameters of metabolic syndrome. ${ }^{33}{ }^{34}$ There have been limited high quality randomized clinical studies utilizing mind-body practices, specifically yoga. A randomized pilot study $(n=41)$ conducted in India compared yoga to a walking control over 8 weeks. The interventions both offered a one day long ( 8 hour) counseling session on lifestyle changes followed by group classes over 8 weeks (3-6 classes per week). Each yoga class also contained 10 minutes of lifestyle education with 32 minutes of active poses per class. The walking group were asked to do 30 minutes of walking over 8 weeks (3-6 classes per week), with no further health education. Participants in both groups attended on average 4 sessions a week during the study period. Mean HOMA-IR and BMI of study participants were lower at baseline than participants in our study. This study reported significant decreases in weight, waist circumference, and BMI between the yoga and walking group. No significant differences between groups were noted in regards to fasting blood glucose and HOMA-IR. No data were reported regarding physical activity and nutritional intake.

Another randomized clinical trial compared restorative yoga versus stretching $(\mathrm{n}=180)$ over 48 weeks among adults with metabolic syndrome [20]. Both groups received 30 minutes of lifestyle education during the first class. The study reported no significant differences in metabolic parameters among participants except that fasting glucose decreased more in the yoga group as compared to stretching. Both groups reported improvements in nutrition and level of physical activity by self-report. An important difference in this study as compared to ours is the use of restorative yoga which does not emphasize any physical activity, and focuses on relaxing aspects of yoga through static postures (hence the name "restorative").

A more recent randomized clinical trial compared yoga to a control group $(\mathrm{n}=182)$ over one year among adults with metabolic syndrome
[22]. Participants in the yoga group were asked to maintain the same dietary intake and physical activity for the duration of the study. The yoga group received yoga three times a week for one year, whereas the control group received usual care. No health education was provided to participants. The study found a reduction in the number of diagnostic components for metabolic syndrome and decreased waist circumference among the yoga group.

Our study is the first study of yoga among participants at risk for metabolic syndrome to implement a standardized, evidence-based lifestyle education (based on the Diabetes Prevention Program). Yoga is a complex behavioral intervention, which provides direct physical activity but may affect cardiometabolic outcomes indirectly through changes in lifestyle (nutrition, other physical activity) and mental health. The intention of our study design was to match the education content in both study groups to isolate and compare the differential effects of yoga. The changes in caloric intake reported by both groups were equally dramatic, perhaps reflecting limitations in questionnaires to assess nutritional intake often subject to underreporting [35]. We utilized actigraphs to collect physical activity levels, though results need to be interpreted with caution due incomplete data collection (only 45 of 67 participants). With these limitations in mind, our preliminary results suggest little difference in physical activity levels or nutrition among groups. This suggests future clinical trials should focus on direct cardiometabolic and psychological effects of yoga rather than lifestyle changes. Continued measurement of physical activity and nutrition are necessary to assess adherence and potential residual confounding.

Our study has several limitations. As mentioned previously, we did not achieve our recruitment goal. Our recruitment mechanism enrolled participants that did not meet all of the standard criteria for metabolic syndrome. Our primary outcome, HOMA-IR, was lower than anticipated in the sample population which also decreased the power to discern differences between groups. Only $70 \%$ of participants provided complete physical activity data, which limited interpretation of this important health behavior. Among individuals in the EDU only group, we did not capture the types of physical activity performed, limiting direct comparison to the yoga group. Finally, our intervention was for 12 weeks, and it is possible a longer intervention or follow-up would have different results.

In conclusion, we successfully implemented a standardized educational intervention with yoga as compared to the well-studied conventional program for adults at risk for cardiometabolic disease in 
Citation: Birdee GS, Ikizler TA, Wheeler A, Bossart C, Wertenbaker D, et al. (2015) Yoga Combined With Health Education for Risk Reduction of Metabolic Syndrome: A Randomized Controlled Pilot Feasibility Study. J Diabetes Metab 6: 588. doi:10.4172/2155-6156.1000588

Page 8 of 8

this pilot randomized trial confirming feasibility. Overall, interventions had no statistically significant differences in cardiometabolic risk reduction as it relates to weight, blood pressure, lipids, and insulin resistance. Participants at higher cardiometabolic risk as assessed by insulin resistance may have further benefits from yoga than conventional education programs. These data provide strong rationale for a larger clinical trial to identify the clinical effectiveness of yoga among adults at high risk for cardiometabolic disease.

\section{Acknowledgement}

This study was funded by Vanderbilt Center for Diabetes Translational Research Pilot Award P30-DK092986. Dr. Gurjeet Birdee is the recipient of Grant Number K23 AT006965 from the National Center for Complementary and Integrative Health. TAI is partly supported by K24 DK62849 from the National Institute of Diabetes, Digestive and Kidney Diseases. SJS is supported by the National Institutes of Health Building Interdisciplinary Research Careers in Women's Health (BIRCWH) Scholar Program (2K12HD043483). This study also received support through the CTSA award No. UL1TR000445 from the Nationa Center for Advancing Translational Sciences. The content is solely the responsibility of the authors and does not necessarily represent the official views of the National Center for Complementary \& Alternative Medicine or National Center for Advancing Translational Sciences or the National Institutes of Health. The authors have no financial disclosures.

\section{References}

1. Ford ES, Li C, Sattar N (2008) Metabolic syndrome and incident diabetes: current state of the evidence. Diabetes Care 31: 1898-1904.

2. Mottillo S, Filion KB, Genest J, Joseph L, Pilote L, et al. (2010) The metabolic syndrome and cardiovascular risk a systematic review and meta-analysis. J Am Coll Cardiol 56: 1113-1132.

3. Alberti KG, Eckel RH, Grundy SM, Zimmet PZ, Cleeman JI, et al. (2009) Harmonizing the metabolic syndrome: a joint interim statement of the International Diabetes Federation Task Force on Epidemiology and Prevention; National Heart, Lung, and Blood Institute; American Heart Association; World Heart Federation; International Atherosclerosis Society; and International Association for the Study of Obesity. Circulation. 120: 1640-1645.

4. Ervin RB (2009) Prevalence of metabolic syndrome among adults 20 years of age and over, by sex, age, race and ethnicity, and body mass index: United States, 2003-2006. Natl Health Stat Report : 1-7.

5. Grundy SM, Cleeman JI, Daniels SR, Donato KA, Eckel RH, et al. (2005) Diagnosis and management of the metabolic syndrome. An American Heart Association/National Heart, Lung, and Blood Institute Scientific Statement. Executive summary. Cardiol Rev. 13: 322-327.

6. Magkos F, Yannakoulia M, Chan JL, Mantzoros CS (2009) Management of the metabolic syndrome and type 2 diabetes through lifestyle modification. Annu Rev Nutr 29: 223-256.

7. Orchard TJ, Temprosa M, Goldberg R, Haffner S, Ratner R, et al. (2005) The effect of metformin and intensive lifestyle intervention on the metabolic syndrome: the Diabetes Prevention Program randomized trial. Ann Intern Med 142: 611-619.

8. Anderssen SA, Carroll S, Urdal P, Holme I (2007) Combined diet and exercise intervention reverses the metabolic syndrome in middle-aged males: results from the Oslo Diet and Exercise Study. Scand J Med Sci Sports 17: 687-695.

9. Fappa E, Yannakoulia M, Pitsavos C, Skoumas I, Valourdou S, et al. (2008) Lifestyle intervention in the management of metabolic syndrome: could we improve adherence issues? Nutrition 24: 286-291.

10. Lutz A, Slagter HA, Dunne JD, Davidson RJ (2008) Attention regulation and monitoring in meditation. Trends Cogn Sci 12: 163-169.

11. Benson $H$ (1975) The relaxation response. New York, NY: Morrow.

12. Barnes PM, Bloom B, Nahin RL (2008) Complementary and alternative medicine use among adults and children: United States, 2007. Natl Health Stat Report : 1-23.

13. Zhang $Y$ (2014) American Adult Yoga Practice: Preliminary Findings from NHIS 2012 Data. The Journal of Alternative and Complementary Medicine. 20: A122-A122.

14. Desikachar T (1999) The heart of yoga: Developing a personal practice: Inner Traditions/Bear \& Co.
15. Clay CC, Lloyd LK, Walker JL, Sharp KR, Pankey RB (2005) The metabolic cost of hatha yoga. J Strength Cond Res 19: 604-610.

16. Ray US, Pathak A, Tomer OS (2011) Hatha yoga practices: energy expenditure, respiratory changes and intensity of exercise. Evid Based Complement Alternat Med 2011: 241294.

17. Mody BS (2011) Acute effects of Surya Namaskar on the cardiovascular \& metabolic system. J Bodyw Mov Ther 15: 343-347.

18. Anderson JG, Taylor AG (2011) The metabolic syndrome and mind-body therapies: a systematic review. J Nutr Metab 2011: 276419

19. Daubenmier J, Kristeller J, Hecht FM, Maninger N, Kuwata M, et al. (2011) Mindfulness Intervention for Stress Eating to Reduce Cortisol and Abdominal Fat among Overweight and Obese Women: An Exploratory Randomized Controlled Study. J Obes 2011: 651936.

20. Kanaya AM, Araneta MR, Pawlowsky SB, Barrett-Connor E, Grady D, et al. (2014) Restorative yoga and metabolic risk factors: the Practicing Restorative Yoga vs. Stretching for the Metabolic Syndrome (PRYSMS) randomized trial. J Diabetes Complications 28: 406-412.

21. McDermott KA, Rao MR, Nagarathna R, Murphy EJ, Burke A, et al. (2014) A yoga intervention for type 2 diabetes risk reduction: a pilot randomized controlled trial. BMC Complement Altern Med 14: 212.

22. Siu PM, Yu AP, Benzie IF, Woo J (2015) Effects of 1-year yoga on cardiovascular risk factors in middle-aged and older adults with metabolic syndrome: a randomized trial. Diabetol Metab Syndr 7: 40.

23. Kramer MK, Kriska AM, Venditti EM, Miller RG, Brooks MM, et al. (2009) Translating the Diabetes Prevention Program: a comprehensive model for prevention training and program delivery. Am J Prev Med 37: 505-511.

24. Dodani S, Fields JZ (2010) Implementation of the fit body and soul, a churchbased life style program for diabetes prevention in high-risk African Americans: a feasibility study. Diabetes Educ 36: 465-472.

25. Kramer MK, McWilliams JR, Chen HY, Siminerio LM (2011) A communitybased diabetes prevention program: evaluation of the group lifestyle balance program delivered by diabetes educators. Diabetes Educ. 37: 659-668.

26. McTigue KM, Conroy MB, Bigi L, Murphy C, McNeil M (2009) Weight loss through living well: translating an effective lifestyle intervention into clinical practice. Diabetes Educ 35: 199-204, 208.

27. Seidel MC, Powell RO, Zgibor JC, Siminerio LM, Piatt GA (2008) Translating the Diabetes Prevention Program into an urban medically underserved community: a nonrandomized prospective intervention study. Diabetes Care. 31: 684-689.

28. Matthews DR, Hosker JP, Rudenski AS, Naylor BA, Treacher DF, et al. (1985) Homeostasis model assessment: insulin resistance and beta-cell function from fasting plasma glucose and insulin concentrations in man. Diabetologia 28 412-419.

29. Block G, Hartman AM, Naughton D (1990) A reduced dietary questionnaire: development and validation. Epidemiology 1: 58-64.

30. Melanson EL Jr, Freedson PS (1995) Validity of the Computer Science and Applications, Inc. (CSA) activity monitor. Med Sci Sports Exerc 27: 934-940.

31. Hendelman D, Miller K, Baggett C, Debold E, Freedson P (2000) Validity of accelerometry for the assessment of moderate intensity physical activity in the field. Med Sci Sports Exerc 32: S442-449.

32. Liu X, Miller YD, Burton NW, Brown WJ (2010) A preliminary study of the effects of Tai Chi and Qigong medical exercise on indicators of metabolic syndrome glycaemic control, health-related quality of life, and psychological health in adults with elevated blood glucose. Br J Sports Med. Aug 44: 704-709.

33. Pattyn N, Cornelissen VA, Eshghi SR, Vanhees L (2013) The effect of exercise on the cardiovascular risk factors constituting the metabolic syndrome: a metaanalysis of controlled trials. Sports Med 43: 121-133.

34. Yamaoka K, Tango T (2012) Effects of lifestyle modification on metabolic syndrome: a systematic review and meta-analysis. BMC Med 10: 138.

35. Molag ML, de Vries JH, Ocké MC, Dagnelie PC, van den Brandt PA, et al. (2007) Design characteristics of food frequency questionnaires in relation to their validity. Am J Epidemiol 166: 1468-1478. 